\title{
Assessing the Likelihood of Obstructive Sleep Apnea among Infertile Population Using STOPBANG and Modified Berlin Questionnaire: A Cross-sectional Study
}

\author{
Meena Andiappan ${ }^{1}$, Narghis Bushra ${ }^{2}$, Damal Chandrasekar Mathangi ${ }^{3}$, Puvithra Thanikachalam ${ }^{4}$
}

\begin{abstract}
Aim and objective: Obstructive sleep apnea (OSA), a prevalent and mostly underdiagnosed chronic condition, has been investigated for its cardiovascular, metabolic, and neurocognitive consequences in recent times. Intermittent hypoxia, a characteristic feature of OSA, is believed to play a key role in the pathogenesis of these consequences by inducing systemic as well as organ-specific oxidative stress. Indeed, oxidative stress has been recognized as a major causative factor of infertility. Provided that OSA provokes oxidative stress, infertility could be envisaged as a potential consequence in patients with severe OSA. The presence and severity of OSA and its associated risk with systemic comorbidities like diabetes, hypertension, and stroke were well established. Whereas the presence of OSA and its relationship with infertility remains elusive. Hence, the study was initiated to evaluate the prevalence of OSA among infertile people attending a tertiary care hospital compared with the general population of the same age group using two questionnaires-Modified Berlin and STOPBANG.

Materials and methods: A sample of 120 subjects in the reproductive age group with a diagnosis of infertility was screened for the presence of OSA using two standard questionnaires-STOPBANG and Modified Berlin questionnaire.

Results: The prevalence of high-risk OSA in infertile subjects was $7.5 \%$ by Modified Berlin and $15 \%$ by STOPBANG questionnaire. Overall predictive values of the employed questionnaire were fair (ROC area under curve) AUC 0.521 for Modified Berlin and 0.516 for STOPBANG. Multivariate logistic regression analysis revealed male gender, snoring, body mass index (BMl) $>25 \mathrm{~kg} / \mathrm{m}^{2}$, and neck circumference $>40 \mathrm{~cm}$ as significant risk factors for moderate to severe sleep apnea among infertile subjects.

Conclusion: There is increased OSA risk among infertile people and hence the clinical suspicion of OSA should be increased among infertile people with the polycystic ovarian syndrome (PCOS), oligozoospermia, hypertension, diabetes, and obesity.

Clinical significance: Identification of OSA risk among infertile people forms a new perspective in the field of reproductive medicine, thereby helping us to achieve a high success rate in infertility treatment.

Keywords: Infertility, Modified Berlin questionnaire, Obstructive sleep apnea, STOPBANG.

International Journal of Infertility and Fetal Medicine (2020): 10.5005/jp-journals-10016-1204
\end{abstract}

\section{INTRODUCTION}

Sleep is a complex neurological state with the restorative and rejuvenating functions of the body and mind. Sleep and endocrine system are intricately linked and losing the homeostasis of one, may exert its deleterious effect on the other and vice versa. Among the various types of sleep disorders, sleep apnea is gaining attention in recent years. Obstructive sleep apnea (OSA) is a chronic disorder, affecting approximately one-third of the adult population, characterized by the triad of hypoxia-reoxygenation, intrathoracic pressure swings, and sleep fragmentation. Obstructive sleep apnea is often an underestimated condition and it has been reported that nearly $13 \%$ of Indians suffer from OSA, of which medical attention is sought by only $4 \%$. Also, the incidence of OSA is threefold higher among men than women. ${ }^{1}$ In Indian studies, OSA prevalence varies from 4.4 to $19.7 \%$ in men and from 2.5 to $7.4 \%$ in women. ${ }^{2}$

Untreated sleep apnea is often associated with high blood pressure, stroke, obesity, and diabetes mellitus, and hence the presence of OSA should be screened for better treatment outcome. ${ }^{3}$ Obstructive sleep apnea is known to negatively influence the reproductive potential of both the gender as evidenced from other studies. Torres et al. ${ }^{4}$ were the first to establish that intermittent hypoxia, a feature of OSA, was associated with local cyclic desaturation of reproductive tissue, leading to diminished antioxidant capacity and subsequently resulting in men's infertility. In addition, it was also found that $10-60 \%$ of OSA subjects develop
${ }^{1-3}$ Department of Physiology, Chettinad Hospital and Research Institute, Kanchipuram, Tamil Nadu, India

${ }^{4}$ Department of Reproductive Medicine, Chettinad Hospital and Research Institute, Kanchipuram, Tamil Nadu, India

Corresponding Author: Meena Andiappan, Department of Physiology, Chettinad Hospital and Research Institute, Kanchipuram, Tamil Nadu, India, Phone: +91 9789843057, e-mail: ameena005@gmail.com

How to cite this article: Andiappan M, Bushra N, Mathangi DC, et al. Assessing the Likelihood of Obstructive Sleep Apnea among Infertile Population Using STOPBANG and Modified Berlin Questionnaire: A Cross-sectional Study. Int J Infertil Fetal Med 2020;11(2):42-47.

Source of support: Nil

Conflict of interest: None

erectile dysfunction, as an effect of endothelial dysfunction signal. ${ }^{5}$ Obstructive sleep apnea also causes suppression of hypothalamogonadal axis, resulting in men hypogonadism. ${ }^{6}$ In a study by Hammoud et al., it was recommended to screen for OSA among men with hypogonadism, erectile dysfunction, and altered sperm parameters and their reproductive potential could be improved by treating the modifiable risk factor-sleep apnea. ${ }^{7}$

Though the association of OSA with sexual dysfunction in men has been elucidated, studies relating the effects of OSA on the sexual function of women are meager. ${ }^{8}$ However, it has been 
reported that untreated OSA negatively impacts women's sexual function. ${ }^{9}$ It has also been observed that obese women with the polycystic ovarian syndrome (PCOS) often have coexisting OSA. ${ }^{10}$ The risk of OSA among PCOS women could be attributed to high androgen levels and visceral obesity. Moreover, untreated OSA is also associated with pregnancy-related complications like gestational hypertension, gestational diabetes, spontaneous abortion, and stillbirth. These adverse outcomes might be attributed to intermittent hypoxia and oxidative stress underlying OSA leading to endothelial dysfunction. ${ }^{11}$ Though obesity is a known firm cause of infertility, the effect of obesity on fertility is confounded to a large extent by OSA, affecting the hormonal profile and regularity of menstrual cycles in women of childbearing age. ${ }^{12}$ These studies indicate that OSA can be an emerging risk factor for infertility. To date, the majority of works have focused on a single cause of infertility like either erectile dysfunction or PCOS and OSA. Hence, our study was initiated to find the risk for OSA among infertile subjects attending a tertiary care hospital in Chennai.

\section{Materials and Methods}

This cross-sectional study was initiated, after obtaining clearance from the Institutional ethics committee. The study was conducted in accordance with the declaration of Helsinki among the infertile subjects attending the Department of Andrology and Reproductive Medicine, Chettinad Hospitals and Research Institute. The study included subjects in the reproductive age group of 25-40 years. The exclusion criteria included the presence of craniofacial abnormalities, history of cranial trauma, chronic pulmonary disease, and recent upper airway surgeries. The sample size of 140 was calculated based on the OSA prevalence in India (9.3\%) with the power of the study being around $80 \%$. Basic anthropometric measures like height, weight, and vital signs were recorded. Additionally, some pertinent information related to the study, such as, type and cause of infertility, were obtained from the subject's case sheet after obtaining their due consent. Those infertile subjects who gave their consent (written) to participate in the study were explained in detail about the study in their regional language. Our study had no controls as it was designed as a cross-sectional study to investigate the prevalence of OSA only in the infertile populations. After obtaining consent, the OSA screening questionnaireSTOPBANG was administered along with their spouse. Though polysomnography is the ideal choice for diagnosing OSA, we chose for STOPBANG questionnaire as the former is time-consuming, expensive, and effort demanding. STOPBANG questionnaire has been validated for use in different populations and patients with varied medical conditions. This questionnaire has four subjective and objective components namely snoring, tiredness, observed apneas, blood pressure, body mass index (BMI), age, gender, and neck circumference. The total score ranges from 0 to 8 . Patients with a score of $0-2$ can be classified as low risk and those with a score of 5-8 at high risk for moderate to severe OSA, respectively. The higher the STOPBANG score, the greater is the probability of moderate and severe sleep apnea. This questionnaire has been authenticated for screening OSA with a high sensitivity of $98 \% .^{13-15}$

Berlin questionnaire validated for use in the western population was modified by AllMS in 2006 for application in the setting of developing countries. It comprises three categories of questions related to the presence and severity of snoring, frequency of daytime sleepiness, and the presence of hypertension. The response to each question is based on a four-point frequency scale [0-never, 1 -rarely (1-2/month), 2-sometimes (1-2/ week), 3-frequently (3-4/week), 4-always (>4/week)]. High risk was defined as persistent symptoms ( $>3$ to 4 times/week) in two or more questions in category 1 and 2 concerned about snoring and daytime sleepiness, respectively, whereas in category III, highrisk OSA was defined as the presence of high blood pressure as per JNC VI (Joint National Commission on Prevention, Detection, Evaluation and Treatment of High Blood Pressure) or BMI $>25 \mathrm{~kg} /$ $\mathrm{m}^{2}$. Subjects qualified as high risk in any two symptom categories will be considered as high risk for OSA. Those who qualified as high risk for only one symptom category and those who denied the presence of persistent symptoms are at low risk of OSA.

The idea of employing two questionnaires in this study is to distinguish the diagnostic accuracy of the two based on their sensitivity and positive predictive value (PPV).

Subjects were grouped based on gender and further subclassification was done according to infertility causes. Data were analyzed using SPSS version 21 and $p$ value $<0.05$ was considered statistically significant.

\section{Statistical Analysis}

Obstructive sleep apnea risk based on the STOPBANG questionnaire was considered as the primary outcome variable. Sociodemographic factors, type of infertility, physical parameters like blood pressure, neck circumference, etc., were considered as explanatory parameters. Descriptive analysis was done by the mean and standard deviation for quantitative variables, frequency, and percentages for categorical variables. The association between various categorical explanatory variables and OSA categories was assessed by cross-tabulation and comparison of proportions. Chi-square test was used to test the statistical significance. The numerical variables were compared across various OSA categories by independent sample $t$-test. $p$ value $<0.05$ was considered as statistically significant. IBM SPSS version 21 was used for statistical analysis.

\section{Results}

Of the total 140 volunteered subjects, only 120 subjects completed the questionnaire (attrition rate-14.3\%). Subjects were grouped based on gender and further subgrouping was based on the type of infertility. Among the 120 infertile subjects (60 male and 60 female), primary infertility was found in 96 subjects, while the remaining 24 had secondary infertility. The mean age of subjects was $31.78 \pm 4.76$ years. Demographic data of the study participants were given in Table 1.

The most common cause of infertility was observed to be oligozoospermia (33.3\%) in male and tubal blockage (16.7\%) in female subjects. The distribution of various causes of infertility among each gender was given below (Figs 1 and 2.)

Table 1: Characteristics of all studied participants

\begin{tabular}{ll}
\hline Age $($ mean \pm SD) (years) & $31.78 \pm 4.76$ \\
Sex $[n(\%)]$ & $60(50)$ \\
$\quad$ Male & $60(50)$ \\
$\quad$ Female & $26.58 \pm 4.61$ \\
Body mass index (mean \pm SD) $\left(\mathrm{kg} / \mathrm{m}^{2}\right)$ & $38.67 \pm 3.9$ \\
Neck circumference $($ mean $\pm \mathrm{SD})(\mathrm{cm})$ & $115.67 \pm 13.08$ \\
Systolic blood pressure $($ mean $\pm \mathrm{SD})(\mathrm{mm} \mathrm{Hg})$ & $73.67 \pm 9.07$ \\
Diastolic blood pressure (mean $\pm \mathrm{SD})(\mathrm{mm} \mathrm{Hg})$ & \\
\hline
\end{tabular}




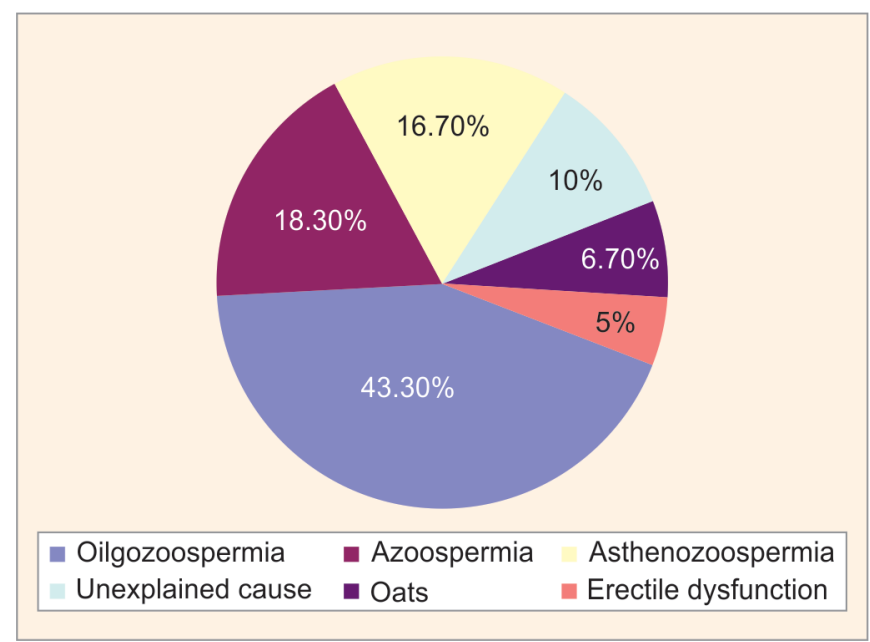

Fig. 1: Distribution of causes of male infertility

Table 2: Comparison of modified Berlin and STOPBANG questionnaire in screening OSA among infertile subjects

\begin{tabular}{lll}
\hline & STOPBANG & Modified Berlin \\
\hline Sensitivity (\%) & 88.9 & 88.9 \\
Specificity (\%) & 78.4 & 79 \\
Positive predictive value (\%) & 91.7 & 95.8 \\
Negative predictive value (\%) & 16.7 & 8.3 \\
$p$ value & $<0.001^{\mathrm{a}}$ & $<0.001^{\mathrm{a}}$ \\
\hline
\end{tabular}

${ }^{\mathrm{a}}$ Both $p$ values are significant $<0.05$

High-risk OSA individuals identified by Modified Berlin and STOPBANG questionnaires were $7.5 \%(n=9)$ and $15 \%(n=18)$, respectively. Among the high-risk individuals, $28.3 \%$ were men and $3.3 \%$ were women.

Though sensitivity of both the questionnaire is equal, specificity and PPV of Modified Berlin is more compared to STOPBANG (Table 2). The difference in sensitivity and specificity of the two questionnaires could be attributed to age, sex, body mass index, and sample size. The significant difference between PPV and sensitivity in determining the likelihood of a test diagnosing a disease is that PPV is dependent on the prevalence of a condition, whereas sensitivity is not. Hence, the sensitivity of a questionnaire is more important in diagnosing a condition followed by PPV.

Neck circumference and blood pressure exhibit a statistically significant difference between high and low risk OSA subjects screened by Modified Berlin (Table 3).

Height, weight, neck circumference, blood pressure, and age demonstrate a statistically significant difference among the OSA risk group screened by STOPBANG (Table 4).

\section{Discussion}

Obstructive sleep apnea, because of its associated comorbidities and under-recognition, poses a major threat to an individual's well-being. The significant finding in our study is $15 \%$ of infertile subjects were at high risk for OSA. None of our study participants were earlier diagnosed with OSA. So, this greater percentage of OSA risk among infertile subjects reflects a low index of suspicion of the health hazards of OSA. Also, our study showed a high risk of OSA among men than women subjects. This could be attributed to the inherent limitation of the STOPBANG scoring system, as it

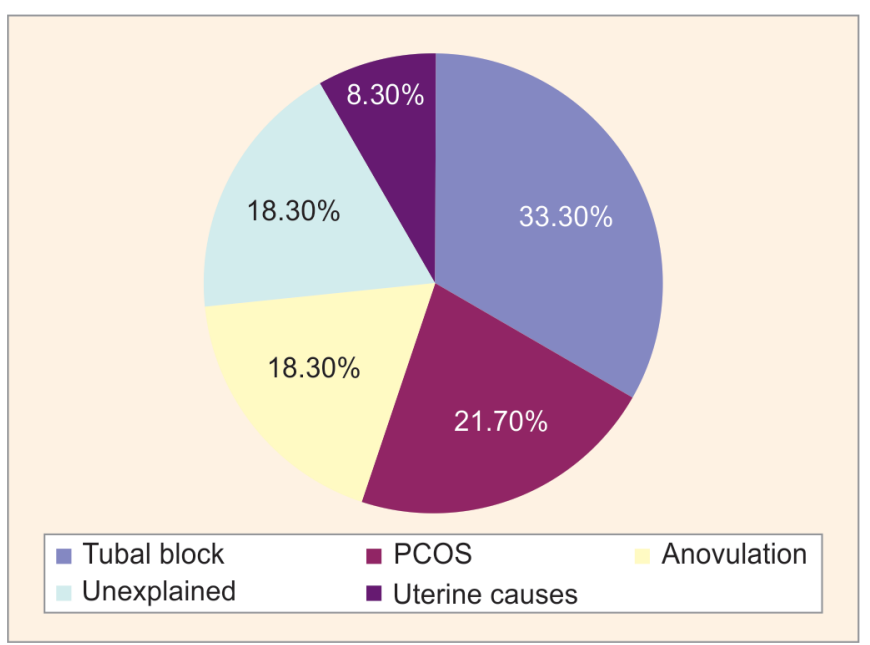

Fig. 2: Distribution of causes of female infertility

Table 3: Comparison of means of anthropometric measures between high- and low-risk OSA subjects screened by modified Berlin questionnaire

\begin{tabular}{|c|c|c|c|}
\hline \multirow[b]{2}{*}{ Parameters } & \multicolumn{2}{|c|}{ Mean $\pm S D$} & \multirow[b]{2}{*}{$p$ value } \\
\hline & Low risk & High risk & \\
\hline Height $(\mathrm{cm})$ & $160.8 \pm 10.5$ & $165.7 \pm 5$ & 0.177 \\
\hline Weight (kg) & $68.3 \pm 15.2$ & $83.6 \pm 14.8$ & 0.005 \\
\hline BMI $\left(\mathrm{kg} / \mathrm{m}^{2}\right)$ & $26.5 \pm 4.7$ & $27.8 \pm 2.2$ & 0.426 \\
\hline Neck circumference $(\mathrm{cm})$ & $38.3 \pm 3.8$ & $43 \pm 2.7$ & $0.000^{\mathrm{a}}$ \\
\hline $\begin{array}{l}\text { Systolic blood pressure } \\
(\mathrm{mm} \mathrm{Hg})\end{array}$ & $114.3 \pm 12.4$ & $132.2 \pm 10$ & $0.000^{\mathrm{a}}$ \\
\hline $\begin{array}{l}\text { Diastolic blood pressure } \\
(\mathrm{mm} \mathrm{Hg})\end{array}$ & $73 \pm 9$ & $82.2 \pm 6.7$ & $0.003^{\mathrm{a}}$ \\
\hline Age (years) & $31.7 \pm 4.8$ & $32.7 \pm 4.3$ & 0.562 \\
\hline
\end{tabular}

a Significant $<0.05$

Table 4: Comparison of means of anthropometric measures between high- and low-risk OSA subjects screened by STOPBANG questionnaire

\begin{tabular}{|c|c|c|c|}
\hline \multirow[b]{2}{*}{ Parameters } & \multicolumn{2}{|c|}{ Mean $\pm S D$} & \multirow[b]{2}{*}{$p$ value } \\
\hline & Low risk & High risk & \\
\hline Height $(\mathrm{cm})$ & $157.3 \pm 9.4$ & $168.8 \pm 8.2$ & $0.000^{\mathrm{a}}$ \\
\hline Weight (kg) & $64.4 \pm 13.3$ & $80.9 \pm 17.3$ & $0.000^{\mathrm{a}}$ \\
\hline BMI $\left(\mathrm{kg} / \mathrm{m}^{2}\right)$ & $26.1 \pm 4$ & $26.9 \pm 3.9$ & 0.296 \\
\hline Neck circumference $(\mathrm{cm})$ & $36.8 \pm 3.5$ & $42.1 \pm 2.0$ & $0.000^{\mathrm{a}}$ \\
\hline $\begin{array}{l}\text { Systolic blood pressure } \\
(\mathrm{mm} \mathrm{Hg})\end{array}$ & $111.9 \pm 12.8$ & $124.4 \pm 12$ & $0.000^{\mathrm{a}}$ \\
\hline $\begin{array}{l}\text { Diastolic blood pressure } \\
(\mathrm{mm} \mathrm{Hg})\end{array}$ & $70.8 \pm 8.5$ & $80 \pm 7.7$ & $0.000^{\mathrm{a}}$ \\
\hline Age (years) & $30.6 \pm 4.7$ & $33.3 \pm 5.1$ & $0.001^{\mathrm{a}}$ \\
\hline
\end{tabular}

${ }^{\text {aSignificant }<0.05}$

gives a point value of 1 to the male gender. So naturally, men tend to have a higher score than women, despite having other features in common. Moreover, women are more unlikely to report their sleep habits and snoring, as they consider it to be a sign of disgrace.

Among the high-risk OSA subjects, most of them had primary infertility. Regardless of OSA risk too, primary infertility was more common in our study population than secondary infertility. These results are in comparison with the study from the urban population 
of Tamil Nadu, which showed $82.4 \%$ of primary infertility and $17.52 \%$ of secondary infertility. ${ }^{16}$

The possible mechanisms by which OSA may predispose to infertility could be explained. Obstructive sleep apnea is characterized by sleep fragmentation, intermittent hypoxia, and oxidative stress. Sleep fragmentation could result in either suppression or augmentation of reproductive hormones, thereby having an impact on successful conception. ${ }^{17,18}$ Obstructive sleep apnea is also associated with hyperactivity of hypothalamopituitary-adrenal (HPA) axis, contributing to insulin resistance. ${ }^{19}$ Insulin resistance is the potential contributing factor to infertility and early miscarriages, among PCOS women in particular. Oxidative stress associated with OSA also remains a detrimental factor to fertility by exerting a negative influence on ovarian function, implantation, and sperm production. It has been hypothesized that increased HPA activity, oxidative stress, and elevated sympathetic activity are detrimental to numerous vital steps in conception. ${ }^{20}$ Though the plausible mechanisms of OSA predisposing to infertility have been explained, there is no concrete evidence for OSA leading to infertility. The presence of OSA and its severity may have an influence over infertility and hence screening for OSA among infertile people may be warranted.

Indeed, a large multicentric study showed a significant improvement in their intimate and sexual relationship after continuous positive airway pressure (CPAP) for 3 months. ${ }^{21}$ It has also been shown that OSA patients with erectile dysfunction responded positively to CPAP treatment. ${ }^{22}$ Though CPAP therapy has a beneficial effect on sexual dysfunction, its role in normalizing testosterone levels remains controversial. ${ }^{23}$ Further CPAP therapy among OSA subjects resulted in a decrease of elevated prolactin levels, which could imply PCOS management. ${ }^{24}$ Obstructive sleep apnea is also known to affect the quality of sexual life, ${ }^{25}$ and CPAP therapy yielded a fruitful result in terms of quality of life. ${ }^{10}$ Hence, the institution of CPAP therapy might help in the reversal of fertility among infertile subjects with OSA.

By quantifying and qualifying the presence of OSA among infertile people, clinicians can be sensitized about OSA as a potential comorbid condition with infertility. Moreover, the institution of OSA treatment along with other modalities of infertility treatment would result in enhanced quality of life at the minimum. If this is going to be the case, OSA treatment may serve to increase the potential for response to fertility treatment and better reproductive outcomes.

The main limitations of the study are a small sample size and a lack of confirmatory OSA diagnosis using overnight polysomnography. Furthermore, there is also a possibility of subjective bias owing to the nature of the study (questionnairebased). The study is also confounded by the effect of obesity which is a common risk factor for infertility and OSA.

By knowing that OSA risk is greater among infertile people, future studies could be done as a case-control study with a large sample size (calculated based on infertility prevalence). Furthermore, the presence of OSA could also be validated using overnight polysomnography and the outcome of fertility treatment assessed independently or in conjunction with treatment for OSA.

\section{Conclusion}

The overall aspect of the study gives an insight into the OSA risk among the infertile populations insisting on the importance of OSA screening in people with infertility. Though identification of high-risk OSA subjects is relatively easy and cost-effective using the questionnaire, the results obtained out of it might not be fruitful as it is associated with subjective bias and the presence of OSA cannot be confirmed. Hence, our findings raise the need for adequately powered studies with appropriate adjusting for confounding variables, large sample size, and polysomnography to truly ascertain OSA as a risk factor for infertility. Furthermore, research is needed to elucidate the causal mechanisms linking OSA and infertility, as a better understanding of the link could lead to better treatment options.

\section{References}

1. Reddy EV, Kadhiravan T, Mishra HK, et al. Prevalence and risk factors of obstructive sleep apnea among middle-aged urban Indians: a community-based study. Sleep Med 2009;10(8):913-918. DOI: 10.1016/j.sleep.2008.08.011.

2. Prasad CN. Obstructive sleep apnea hypopnea syndrome - Indian scenario. Persp Med Res 2013;1(1):22-25.

3. Pinto J, Ribeiro D, Cavallini A, et al. Comorbidities associated with obstructive sleep apnea: a retrospective study. Int Arch Otorhinolaryngol 2016;20(2):145-150. DOI: 10.1055/s-00361579546.

4. Torres M, Laguna-Barraza R, Dalmases M, et al. Male fertility is reduced by chronic intermittent hypoxia mimicking sleep apnea in mice. Sleep [Internet]. 2014 [cited 2016 Mar 15]

5. Hirotsu C, Tufik S, Levy Andersen M. Sleep apnea as a potential threat to reproduction. Sleep 2014;37(11):1731-1732. DOI: 10.5665/ sleep.4158.

6. Luboshitzky R, Aviv A, Hefetz A, et al. Decreased pituitary-gonadal secretion in men with obstructive sleep apnea. J Clin Endocrinol Metab 2002;87(7):3394-3398. DOI: 10.1210/jcem.87.7.8663.

7. Hammoud AO, Carrell DT, Gibson M, et al. Updates on the relation of weight excess and reproductive function in men: sleep apnea as a new area of interest. Asian J Androl 2012;14(1):77-81. DOI: 10.1038/ aja.2011.64.

8. Tamanna S, Geraci SA. Major sleep disorders among women: (women's health series). South Med J 2013;106(8):470-478. DOI: 10.1097/SMJ.0b013e3182a15af5.

9. Petersen $M$, Kristensen $E$, Berg $S$, et al. Sexual function in female patients with obstructive sleep apnea. J Sex Med 2011;8(9):25602568. DOI: 10.1111/j.1743-6109.2011.02358.x.

10. Kapoor S. The close relationship between sleep disorders and reproductive dysfunction in endocrinology. Front Endocrinol (Lausanne) Frontiers Media SA 2012;3:117. DOI: 10.3389/ fendo.2012.00117.

11. Lee EK, Gutcher ST, Douglass AB. Is sleep-disordered breathing associated with miscarriages? An emerging hypothesis. Med Hypotheses 2014;82(4):481-485. DOI: 10.1016/j.mehy.2014.01.031.

12. Champagne KA, John Kimoff R, Barriga PC, et al. Sleep disordered breathing in women of childbearing age and during pregnancy. Indian J Med Res 2010;131:285-301.

13. El-Sayed I. Comparison of four sleep questionnaires for screening obstructive sleep apnea. Egypt J Chest Dis Tuberc 2012;61(4):433-441. DOI: 10.1016/j.ejcdt.2012.07.003.

14. Nagappa M, Liao P, Wong J, et al. Validation of the STOP-bang questionnaire as a screening tool for obstructive sleep apnea among different populations: a systematic review and meta-analysis. PLoS ONE 2015;10(12):1371. DOI: 10.1371/journal.pone.0143697.

15. Chung F, Subramanyam R, Liao P, et al. High STOP-bang score indicates a high probability of obstructive sleep apnoea. Br J Anaesth. Oxford University Press; 2012;108(5):768-775.

16. Chaudhry P, Shrivastava D, Jaiswal A. To study the prevelance of etiological factors resposible for subfertility and its correlation with clinical profile in rural India. Indian J Appl Res 2016;5(10):731-735.

17. Goswami R. Endocrine and metabolic aspects of OSA. Ann Natl Acad Med Sci 2013;49(3\&4):122-131. 
18. Kloss JD, Perlis ML, Zamzow JA, et al. Sleep, sleep disturbance, and fertility in women. Sleep Med Rev 2015;22:78-87. DOI: 10.1016/j. smrv.2014.10.005.

19. Buckley TM, Schatzberg AF. On the interactions of the hypothalamicpituitary-adrenal (HPA) axis and sleep: normal HPA axis activity and circadian rhythm, exemplary sleep disorders. J Clin Endocrinol Metab Endocrine Society 2005;90(5):3106-3114. DOI: 10.1210/jc.2004-1056.

20. Goldstein CA, Smith YR. Sleep, circadian rhythms, and fertility. Curr Sleep Med Reports, Springer International Publishing 2016;2(4):206217. DOI: 10.1007/s40675-016-0057-9.

21. Reishtein JL, Maislin G, Weaver TE. Outcome of CPAP treatment on intimate and sexual relationships in men with obstructive sleep apnea. J Clin Sleep Med Am Acad Sleep Med 2010;6(3):221-226. DOI: $10.5664 / j c s m .27817$.
22. Taskin U, Yigit $\mathrm{O}$, Acioglu $\mathrm{E}$, et al. Erectile dysfunction in severe sleep apnea patients and response to CPAP. Int J Impot Res 2009;22(10): 134-139.

23. Zhang X-B, Jiang X-T, Du Y-P, et al. Efficacy of continuous positive airway pressure on testosterone in men with obstructive sleep apnea: a meta-analysis. PLoS One Pub Libr Sci 2014;9(12):e115033. DOI: 10.1371/journal.pone.0115033.

24. Nitsche K, Ehrmann DA. Obstructive sleep apnea and metabolic dysfunction in polycystic ovary syndrome. Best Pract Res Clin Endocrinol Metab 2010;24(5):717-730. DOI: 10.1016/j. beem.2010.08.001.

25. Hammoud AO, Walker JM, Gibson M, et al. Sleep apnea, reproductive hormones and quality of sexual life in severely obese men. Obesity 2011;19(6):1118-1123. DOI: 10.1038/oby.2010.344. 


\section{Annexure I}

\section{Screening Questions}

1. Do you snore?

2. Do you feel tired after waking up from sleep?

3. Do you feel you are obese?

4. Are you a hypertensive?

\section{Annexure II}

Modified Berlin questionnaire (used at AlIMS, New Delhi)

\section{Category 1}

1. Do you snore?

Yes

No

Don't know

2. Your snoring is

Slightly louder than breathing

As loud as talking

Louder than talking

Very loud can be heard in adjacent rooms

3. How often do you snore?

Nearly every day

3-4 times a week

1-2 times a week

$1-2$ times a month

Never or nearly never

4. Has your snoring ever bothered other people?

Yes

No

5. Has anyone noticed that you quit breathing during your sleep?

If yes, how frequently?

Nearly every day

3-4 times a week

1-2 times a week

$1-2$ times a month

Never or nearly never

6. Do you choke while you are sleeping? If yes, how frequently? Nearly every day

3-4 times a week

$1-2$ times a week

\section{1-2 times a month}

Never or nearly never

\section{Category 2}

1. How often do your feel tired or fatigued after your sleep?

Nearly every day

3-4 times a week

1-2 times a week

1-2 times a month

Never or nearly never

2. During your wake time do you feel tired, fatigued, or not up to at par? If yes, how frequently?

Nearly every day

3-4 times a week

1-2 times a week

1-2 times a month

Never or nearly never

3. Have you ever fallen asleep while waiting in a line to meet your doctor? If yes, how frequently?

Nearly in all visits

In 3-4 visits

In 1-2 visits

Never or nearly never

4. Have you ever fallen asleep while watching television at your home during the daytime? If yes, how frequently?

Nearly every day

3-4 times a week

1-2 times a week

1-2 times a month

Never or nearly never

5. Have you ever fallen asleep while waiting in a line to pay your electricity and telephone bills? If yes, how frequently?

Nearly every visit

In 3-4 visits

In 1-2 visits

Never or nearly never

\section{Category 3}

1. Do you have high blood pressure?

Yes

No

Don't know 